Self-confidence also improved (pre-evaluation score: $65.4 \pm$ 17.6 , post-evaluation score: $82.0 \pm 9.4, p$-value $<0.001)$, with all groups reaching statistical significance.

Discussion: This course creates real operative situations in a standardized fashion that improves knowledge and operative confidence for trauma operations, which may be of great benefit in disaster medicine training.

Prehosp Disaster Med 2011;26(Suppl. 1):s117-s118

doi:10.1017/S1049023X11003918

\section{(P1-60) Clinico-Epidemiological Pattern and Management Options in Patients with Ocular Injuries in the Emergency Room of a Rural Hospital \\ S. Baisakbiya, ${ }^{1}$ M. Bhatt, ${ }^{2}$ A. Agrawal ${ }^{3}$ \\ 1. Ophthalmology, Mullana (Ambala), India \\ 2. Anesthesiology, Ambala, India \\ 3. Neurosurgery, Ambala, India}

Aims and Objectives: Successful patient outcomes in the setting of ocular emergencies depend on correct recognition and assessment as well as appropriate initial management. The purpose of this study is to describe the clinico-epidemiological findings and management options in patients with ocular injuries in the emergency room of a rural hospital.

Material and Methods: In this retrospective review the records of patients who were treated for ocular trauma from June 2010 to December 2010 in the emergency room of MM Institute of Medical Sciences and Research, Mullana (Ambala) were reviewed. The following data for all patients were recorded: age, sex, date and time of injury, involved eye, circumstance and mechanism of injury, initial visual acuity, details of appropriate investigation, immediate management and outcome.

Results: A total 46 patients were included in the study. Young adult male patients were more commonly involved. Most of the patients presented within 12 hours of injury. Left eye was involved in 14 patients; right in 12 and 10 patients had injuries to both eyes. Most common mode of injury was mechanical. The patients who sustained bilateral ocular injuries were due to chemical burns ( 7 cases) and electrocution (3 cases). Most of the patients were managed conservatively. The surgical treatment offered were repair of corneal tears, removal of foreign bodies. The visual outcome was excellent in most of the patients.

Conclusions: Ocular injuries can have wide range of etiological factors and presentation and assessment of ocular emergencies can be made difficult by a lack of sophisticated facilities. However, a concise patient history, general observation and basic ocular tests can lead to a firm diagnosis and thereby appropriate management.

Prehosp Disaster Med 2011;26(Suppl. 1):s118

doi:10.1017/S1049023X1100392X

(P1-61) Nursing Education for Disaster Management J. Boone, ${ }^{1}$ D. Moore 2

1. Director of Global Studies, Ontario, United States of America

2. Nursing, Coast Mesa, United States of America

In Southern California in 2010, there were 76 declared disasters of which the majority were in Southern California, for this reason and at the request of our clinical partners, when
West Coast University (WCU) started its nursing program in 2008, it recognized the need to have a course in disaster management. With nursing being the largest of the health professions, the American Association of Colleges of Nursing (AACN) recognized the need to incorporate disaster nursing in its new Baccalaureate Nursing standards. The Disaster Management course was developed in concert with other parts of the curriculum such as Leadership, Physical Assessment, Critical Care courses to help students focus their assessment and intervention skills to prepare them to be future responders. The disaster management course is delivered in both didactic and hands on format including online Federal Emergency Management Agency (FEMA) modules, disaster drills, guest lecturers and field trips. Students are also introduced to many volunteer opportunities and are trained to be disaster ready once they are needed. Students are excited and ready to volunteer at various organizations before graduation such as the American Red Cross, the Medical Volunteer Program and the Disaster Medical Assistance Teams. Since WCU has new campuses, the students have been instrumental in implementing a new disaster plan for our facilities, including developing flyers, videos and conducting disaster drills. We have received feedback from our clinical partners that our students are better prepared than their current emergency staff in regards to disaster management and to that end we plan to work with our clinical partners to translate our class into an online course so their staff can be trained on disaster management.

Prehosp Disaster Med 2011;26(Suppl. 1):s118 doi:10.1017/S1049023X11003931

(P1-62) The Risk Factors for Difficult or Failed Airway: A Prosepctive Cohor Study

C. Hsu

Department of Emergency Medicine, Taipei, Taiwan

The risk factors for difficult airway or failed airway: a prospective cohort study Airway management is always the first priority and time-treasures in critical ill-patients. Improper management of difficult airway or resultant fail airway would bring poor prognosis to patients. We investigated the risk factors of difficult or fail airway from the multiple dimension of factors including patients, healthcare and airway devices. We enrolled 252 intubated patients, including 37 trauma patients, 55 patients $(22 \%)$ with difficult airway, and 22 patients $(8.7 \%)$ with fail airway. In analysis of risk factors of difficult airway, factors including obesity, short neck or thickness of soft tissue, facial deformities and oral-nasal bleeding have positive association with fail airway, but the seniority of healthcare providers had no effect. However, experienced healthcare providers have more success rate after the occurrence of fail airway. The most complications of fail airway include airway trauma and hypoxia. As compared with non-trauma patients, trauma patients have more episodes of fail airway, difficult airway, and use of RSI, rescue airway for fail airway, airway trauma and vomiting. Therefore, it is necessary to establish an easy and safe standard guideline in daily practice of difficult and urgent airway management for healthcare providers.

Prehosp Disaster Med 2011;26(Suppl. 1):s118

doi:10.1017/S1049023X11003943 